\title{
Drooling in Parkinson's Disease: A Multifactorial Symptom
}

\author{
Jalal Bakhtiyari ${ }^{1}$, Azar Mehri ${ }^{2,}$, Saman Maroufizadeh ${ }^{3}$ and Hanif Amanian ${ }^{2}$ \\ ${ }^{1}$ Neuromuscular Rehabilitation Research Center, Semnan University of Medical Sciences, Semnan, Iran \\ ${ }^{2}$ Department of Speech Therapy, School of Rehabilitation, Tehran University of Medical Sciences, Tehran, Iran \\ ${ }^{3}$ School of Nursing and Midwifery, Guilan University of Medical Sciences, Rasht, Iran \\ *Corresponding author: Department of Speech Therapy, School of Rehabilitation, Tehran University of Medical Sciences, Tehran, Iran. Email: mehri@tums.ac.ir
}

Received 2019 November 01; Revised 2020 January 11; Accepted 2020 February 05.

\begin{abstract}
Background: Drooling or Sialorrhoea is considered as a common non-motor symptom in patients with Parkinson's disease (PD), however the mechanism of drooling in PD is unknown.

Objectives: The aim of this study was to evaluate the impacts of dysphagia and cognitive function on drooling in PD.

Methods: Eighty-five patients with PD participated in this cross-sectional study. After diagnosis of PD, its severity was determined by the Hoehn and Yahr (HY) scale, which is consisted of five stages and explains the progression symptoms of PD. Evaluation of drooling was done by the Drooling Severity and Frequency scale (DSFS). Dysphagia was diagnosed using the Persian version of Northwestern dysphagia patient check sheet (NDPCS) and cognitive function was assessed through the Persian version of Mini-Mental State examination (MMSE). Data analysis was done with SPSS 22. All analyses were two-tailed tests and a P value of less than 0.05 was considered as statistically significant.

Results: The mean total DSFS score was $3.96 \pm 1.65$, and using a cut-off point of two, the prevalence of drooling was $70.6 \%(n=60)$ in PD patients. In adjusted analysis, the low MMSE score, high HY score, and dysphagia were all associated with drooling.

Conclusions: Although concurrence of dysphagia and drooling is possible in PD, we should also consider cognitive impairments as an important factor in the occurrence of drooling. In addition, further studies are needed to investigate the impact of cognitive domains on drooling in Parkinson's patients.
\end{abstract}

Keywords: Parkinson's Disease, Sialorrhoea, Cognitive Dysfunction, Deglutition Disorders

\section{Background}

Parkinson's disease (PD) is a degenerative disorder of the central nervous system (1). Motor and non-motor symptoms have been identified in PD. Resting tremor, rigidity, bradykinesia, and postural instability are motor symptoms or abnormal movements (1). In addition, nonmotor symptoms have been recognized as an important part of PD, such as cognitive impairment, depression, anxiety, sleep disorders, drooling, pain, and constipation $(2,3)$.

Drooling or Sialorrhoea is considered as a common non-motor symptom in patients with $\mathrm{PD}(4)$.

It is not possible to determine the exact prevalence of drooling in patients with PD, due to the lack of definite definition as well as standard tool for its evaluation. In general, according to the results available studies, the prevalence of drooling in PD has reported between 10\% and 84\% (5).

Evaluation and management of drooling in PD patients is of great importance in the therapeutic and rehabilitation programs of these patients due to its biopsychosocial complications. Inflammatory rash skin around the mouth (perioral dermatitis), saliva aspiration, the increased probability of pulmonary infection, speech disturbance, eating difficulties, and also the decreased quality of life in the patients and their caregivers are announced as complications of drooling (6).

A proper recognition of the etiology and pathophysiology of drooling in PD is essential to provide appropriate management in this group of patients. However, the exact mechanism of drooling in PD is still unknown $(4,6,7)$.

In some studies, it has hypothesized that the excessive salivation and dysphagia are the main causes of drooling in $\operatorname{PD}(7,8)$. However, other studies have shown that salivary production in PD is even lower than that of the control group; thus, it cannot be the cause of drooling in these patients $(8,9)$. Oropharyngeal dysphagia is another component associated with drooling in PD (10). Oropharyngeal dysphagia in PD is due to muscle rigidity, bradykinesia of oral and pharyngeal muscles, and their poor control (11). According to various studies, oropharyngeal dysphagia is regarded as one of the important factors in the occurrence of drooling in PD (12). Studies have shown that although 
dysphagia and drooling are common in PD, they are poorly correlated in this condition. For example, Nobrega et al., in a study demonstrated that dysphagia alone cannot be considered as drooling and other factors should be considered, as well $(11,13)$.

Mild cognitive impairment is one of the most prevalent non-motor symptoms in PD, as it is experienced by approximately $20 \%-50 \%$ of people with PD and in the advanced stages of the disease; more than $80 \%$ of patients more develop severe cognitive impairments and eventually dementia. Studies have shown that executive functions, attention, and recall tasks are the most damaging aspects of cognition (13).

Several studies have revealed that there is a relationship between dysphagia and cognitive impairment (14-18). For examples, Yang Jo et al. (17), in a study indicated that visual attention and executive function impairment may affect oral phase of swallowing in stroke patients. In addition, another study indicated that (18) executive function impairment in the elderly can affect their motor planning of swallowing (19).

\section{Objectives}

Therefore, according to these studies, the purpose of the present study was to determine the effects of cognitive impairment and swallowing disorders on drooling in PD patients.

\section{Methods}

\subsection{Participants and Study Design}

Eighty-five patients with PD participated in this crosssectional study. Patients were selected from the neurology wards of Tehran University hospitals, between August 2014 and September 2015. Inclusion criteria included the age between 50 and 65 years old, clinical diagnosis of PD confirmed by a neurologist and diagnosis based on the United Kingdom Parkinson's Disease Society Brain Bank (UKPDSBB) clinical diagnostic criteria (20), no history of other neurological diseases, no hearing or visual impairments, no use of antipsychotic drugs, use of levodopa as the main drug, and patients in the "on" state. All participants in this study were with distinct diurnal drooling complaints. All assessments were administrated for patients between 9 AM and 16 PM.

After diagnosis of PD, its severity was determinate by the Modified Hoehn and Yahr ( $\mathrm{H}$ and $\mathrm{Y}$ ) scale. This scale consists of five stages and explains the progression symptoms of PD. In this scale, stage 1, unilateral involvement only and 1/5: unilateral and axial involvement; stage 2, bilateral involvement without impairment of balance and 2.5: mild bilateral disease with recovery on pull test; stage 3 , mild to moderate bilateral disease; some postural instability; physically independent; stage 4, severe disability, still able to walk or stand unassisted; and stage 5, wheelchair bound or bedridden unless aided. Based on our observation of the patient's condition, the severity and progression of the disease were determined by the $\mathrm{H}$ and $\mathrm{Y}$ scale.

Evaluation of drooling was done by the Drooling Severity and Frequency scale (DSFS) (21), which is a subjective tool for assessment of drooling in PD and evaluates severity (1 - 5 scores) and frequency (1 - 4 scores) of drooling. Score one indicates dry (never drools), and scores five and four represent profuse and constant drooling. Determination of severity and frequency of the drooling is based on questions from patients.

Dysphagia was diagnosed using the Persian version of Northwestern dysphagia patient check sheet (NDPCS) (22). The NDPCS consists of twenty-eight components divided into five sections. Medical history, behavioral variables, gross motor function, oral motor test, and observations during trial swallow. This test has a dichotomy scoring system (yes and no). The three sections were based on observation and questioning of the patient and were scored based on the observation of the symptom. For assessment of the Oromotor section we observed oral, pharyngeal, laryngeal structure, and function. The last section includes observation of any signs of dysphagia during trial swallows with types of different food consistency (solid, semi-solid and liquid). The total score for patients is calculated from the total number of yes or abnormal items. Based on the score obtained by each patient are diagnosed four main symptoms of dysphagia (aspiration, oral phase disorders, delay of the pharyngeal phase, and pharyngeal phase disorders).

Cognitive function was assessed by the Persian version of the mini mental state examination(MMSE)(23), the total score of (MMSE) was 30 and cutoff score was 23.

\subsection{Ethical Considerations}

This study was approved by the Ethics Committee of Tehran University of Medical Sciences and all patients completed the informed consent form.

\subsection{Instruments}

Drooling Severity and Frequency Scale (DSFS): DSFS is a subjective method for measuring the severity and frequency of drooling. Using this scale, severity and frequency of drooling is scored in patients or caregivers. In DSFS, drooling severity is scored from one to five, whereas its frequency is scored between one and four. 
Northwestern Dysphagia Patient Check Sheet(NDPCS): NDPCS is a scale for assessment of dysphagia. This test consists of five parts and 28 items with a dichotomous scoring system (normal or safe and abnormal or unsafe). In this study, we used the Persian version of NDPCS, which has shown with appropriate validity and reliability.

Mini-Mental State Examination (MMSE): The MMSE or Folstein test is a method that is used for grading the cognitive state of patients in the clinical and research studies. The questionnaire consists of 20 questions and a total score of 30 points, in which a score of less than 23 indicates the probability of cognitive impairment.

\subsection{Statistical Analysis}

In this study, continuous variables were presented as mean \pm standard deviation (SD) and categorical variables were presented as number (percentage). In the bivariate analysis, the relationships between demographic/clinical characteristics and the DSFS scores were examined via Pearson's correlation coefficient and independent $t$-test. In multivariate analysis, to identify the factor associated with drooling in patients with PD, multiple regression models were used. Data analysis was done with IBM SPSS Statistics for Windows, Version 22.0 (IBM Crop., Armonk, NY, USA). All analyses were two-tailed tests and a P value of less than 0.05 was considered as statistically significant.

\section{Results}

\subsection{Participants' Characteristics}

Table 1 outlines the demographic and clinical characteristics of the 85 patients with PD ( 68 men and 17 women). The mean age and disease duration of the patients were $59.40 \pm 4.79$ and $5.81 \pm 3.68$ years, respectively. Half of the patients had dysphagia and $56.5 \%$ were universityeducated.

The mean MMSE and HY scores were $23.89 \pm 4.81$ and $2.35 \pm 0.86$, respectively. The mean total DSFS score was $3.96 \pm 1.65$, and using a cut-off point of 2 , the prevalence of drooling was $70.6 \%(n=60)$ in PD patients.

\subsection{Bivariate Analysis}

According to the Pearson correlation coefficients, DSFS score was not correlated with age $(\mathrm{r}=-0.105, \mathrm{P}=0.337)$ and duration of disease $(r=0.037, P=0.734)$ (Table 2). There was also no significant correlation between DSFS scores and gender $(\mathrm{P}=0.296)$ and level of education $(\mathrm{P}=0.078)$. Patients who had dysphagia ( $4.98 \pm 1.50)$ obtained significantly higher DSFS scores than those without dysphagia $(2.93 \pm 1.04)$. The DSFS score was negatively correlated with MMSE $(r=-0.627, \mathrm{P}<0.001)$ and positively correlated with HY scores $(\mathrm{B}=0.617, \mathrm{P}<0.001)$.

\begin{tabular}{|c|c|}
\hline & Values \\
\hline Age, $y$ & $59.40 \pm 4.79$ \\
\hline \multicolumn{2}{|l|}{ Sex } \\
\hline Male & $68(80.0)$ \\
\hline Female & $17(20.0)$ \\
\hline \multicolumn{2}{|l|}{ Educational level } \\
\hline Primary/secondary & $37(43.5)$ \\
\hline University & $48(56.5)$ \\
\hline Duration of disease, $y$ & $5.81 \pm 3.68$ \\
\hline \multicolumn{2}{|l|}{ Dysphagia } \\
\hline No & $42(49.4)$ \\
\hline Yes & $43(50.6)$ \\
\hline MMSE & $23.89 \pm 4.81$ \\
\hline HY scale & $2.35 \pm 0.86$ \\
\hline
\end{tabular}

Abbreviations: HY scale, Hoehn and Yahr scale; MMSE, mini mental state exam ination; SD, standard deviation.

${ }^{\mathrm{a}}$ Values are expressed as mean $\pm \mathrm{SD}$ or No. (\%).

Table 2. Relationship Between Drooling and Demographic/Clinical Characteristics in Patients with PD Using Bivariate Analysis

\begin{tabular}{|c|c|c|}
\hline & Mean \pm SD or $\mathbf{r}$ & $\mathbf{P}$ \\
\hline Age & -0.105 & 0.337 \\
\hline Sex & & 0.296 \\
\hline Male & $4.06 \pm 1.75$ & \\
\hline Female & $3.59 \pm 1.12$ & \\
\hline Education & & 0.078 \\
\hline Academic & $4.32 \pm 1.90$ & \\
\hline Non-academic & $3.69 \pm 1.39$ & \\
\hline Duration of disease & 0.037 & 0.734 \\
\hline Dysphagia & & $<0.001$ \\
\hline No & $2.93 \pm 1.04$ & \\
\hline Yes & $4.98 \pm 1.50$ & \\
\hline MMSE & -0.627 & $<0.001$ \\
\hline HY scale & 0.617 & $<0.001$ \\
\hline
\end{tabular}

Abbreviations: r, Pearson correlation coefficient; SD, standard deviation.

\subsection{Multivariate Analysis}

According to the multiple linear regression analysis, patients with dysphagia showed higher DSFS scores than patients without dysphagia $(\mathrm{B}=0.972, \mathrm{P}<0.001)$. The DSFS score was negatively associated with MMSE $(\mathrm{B}=-0.164, \mathrm{P}<$ $0.001)$ and positively associated with HY scores $(B=0.565$, $\mathrm{P}<0.001)$. Consistent with univariate analyses, there were no significant relationship between DSFS score and age $(\mathrm{P}=$ 
0.447), gender $(\mathrm{P}=0.903)$, education $(\mathrm{P}=0.142)$ and disease duration $(\mathrm{P}=0.465)$.

\section{Discussion}

The main finding of this study was a relationship between cognitive impairments and drooling in PD, so that the severity of drooling in terms of DSFS score was negatively associated with MMSE scores. This result confirmed the main hypothesis of this study. Another finding of this study was the association between swallowing disorder and drooling in PD.

Previous studies on drooling have reported a direct correlation between the severity of dysphagia and the severity of drooling $(4,5,11)$. However, in their studies it has demonstrated that there is no relationship between dysphagia and drooling, thus, more than one-third of patients with symptoms of dysphagia had no symptoms of drooling (24). Such discrepancy between the findings of different studies can be due to the used methods for evaluation of swallowing disorder and the number and type of samples. In some studies, only patients with severe symptoms of drooling were included, however, in other studies, cases with moderate to severe symptoms were studied $(5,11,25)$.

Cognitive impairment, as one of the non-motor symptoms, is a common complication in PD and also attention and frontal-executive functions compared to other cognitive domains are more involved in $\operatorname{PD}(14,26)$. Impairments affected some aspects of cognitive function are caused due to brain damages, which affect the oral phase of swallowing (17). Swallowing consists of four phases: preparatory oral stage, transit oral phase, pharyngeal phase, and esophageal phase. The first two stages are voluntary and the other two phases are involuntary. Drooling is known as a symptom of impaired oral phase. Cognition also affects voluntary activities more than involuntary and reflexive activities and any impairment in cognition causes damage to the voluntary aspects of an activity, such as swallowing (26-28).

The management of drooling in PD is important due to its medical and psychosocial complaints for patients and their families. However, like other disorders, knowledge of pathophysiology helps clinicians understand, apply, and develop appropriate treatment plans in their practice. Unfortunately, the precise etiology and pathophysiology of drooling in PD have not fully understood. Many studies have suggested some factors that potentially contribute in drooling in PD, these factors includes, unintentional mouth opening and a flexed head posture $(5,29)$ increased salivary flow rate (30), and dopamine deficiency (4).
Accordingly, the findings of this study cognitive impairments and dysphagia can relate to drooling in PD. Dysphagia causes drooling due to reduced ability to clear saliva from the mouth (31). The main cause of dysphagia in PD is bradykinesia, which results in reduced tongue and lip control, difficulty with mastication (10). As the findings of this study have been indicated in other studies, the severity of dysphagia is related to the severity of drooling in PD (11, 32). However, the relation between cognitive impairment and drooling is still not fully understood. Rana et al. (27), in their study, found that drooling is related to dementia in Parkinson's Disease. Given that cognition encompasses several aspects, (attention, memory and working memory, problem solving and decision making etc.) it is unclear, which aspect of cognition relates to drooling. Kalf et al. (21), in their study indicated that severity of drooling has increased when a person engaged in a concurrent distracting activity, also Reynolds et al. (33), demonstrated that divided attention effects on frequency of saliva swallows and drooling. Further studies are needed to precisely determine the role of cognitive domains in saliva control.

\subsection{Conclusions}

Our data suggested that the cause of drooling in PD is multifactorial and for management of drooling in PD, we should consider all factors such as swallowing problems, cognition impairment and oral motor dysfunction. Also further studies are needed to investigate the impact of cognitive domains on drooling in Parkinson's patients.

\section{Acknowledgments}

The authors are thankful to the patients with PD, their family members for their participation in this study, and also the staff of the Neurology Wards of Tehran University of Medical Sciences, Tehran, Iran for their cooperation.

\section{Footnotes}

Authors' Contribution: Jalal Bakhtiyari did data interpretation and manuscript writing. Azar Mehri did study design, conception, and manuscript writing. Saman Maroufizadeh did data analysis and interpretation. Hanif Amanian did acquisition of data. All authors approved the final version of the article for submission.

Conflict of Interests: The authors declare that they have no conflicts of interests.

Ethical Approval: This study was approved by the Ethics Committee of Tehran University of Medical Sciences.

Funding/Support: This study was founded by the Tehran University of Medical Sciences, Tehran, Iran. 
Informed Consent: All patients completed the informed consent form

\section{References}

1. Lang AE, Lozano AM. Parkinson's disease. N. Engl. J. Med. 1998;339(16):1130-43.

2. Stacy M. Nonmotor symptoms in Parkinson's disease. Int J Neurosci. 2011;121 Suppl 2:9-17. doi: 10.3109/00207454.2011.620196. [PubMed: 22035025].

3. Pfeiffer RF. Non-motor symptoms in Parkinson's disease. Parkinsonism Relat Disord. 2016;22 Suppl 1:S119-22. doi: 10.1016/j.parkreldis.2015.09.004. [PubMed: 26372623].

4. Srivanitchapoom P, Pandey S, Hallett M. Drooling in Parkinson's disease: A review. Parkinsonism Relat Disord. 2014;20(11):1109-18. doi: 10.1016/j.parkreldis.2014.08.013. [PubMed: 25200111]. [PubMed Central: PMC4252747].

5. Kalf JG, Munneke M, van den Engel-Hoek L, de Swart BJ, Borm GF, Bloem BR, et al. Pathophysiology of diurnal drooling in Parkinson's disease. Mov Disord. 2011;26(9):1670-6. doi: 10.1002/mds.23720. [PubMed: 21484876].

6. Kalf JG, Bloem BR, Munneke M. Diurnal and nocturnal drooling in Parkinson's disease. J Neurol. 2012;259(1):119-23. doi: 10.1007/s00415011-6138-2. [PubMed: 21698387]. [PubMed Central: PMC3251785].

7. Jost WH. The option of sonographic guidance in Botulinum toxin injection for drooling in Parkinson's disease. J Neural Transm (Vienna). 2016;123(1):51-5. doi: 10.1007/s00702-015-1416-2. [PubMed: 26138438].

8. Bagheri H, Damase-Michel C, Lapeyre-Mestre M, Cismondo S, O'Connell D, Senard JM. A study of salivary secretion in Parkinson's disease. Clin Neuropharmacol.1999;22(4):213-5.

9. Proulx M, de Courval FP, Wiseman MA, Panisset M. Salivary production in Parkinson's disease. Mov Disord. 2005;20(2):204-7. doi: 10.1002/mds.20189. [PubMed:15389996].

10. Umemoto G, Tsuboi Y, Kitashima A, Furuya H, Kikuta T. Impaired food transportation in Parkinson's disease related to lingual bradykinesia. Dysphagia. 2011;26(3):250-5. doi: 10.1007/s00455-010-9296-y. [PubMed: 20803220].

11. Nobrega AC, Rodrigues B, Torres AC, Scarpel RD, Neves CA, Melo A. Is drooling secondary to a swallowing disorder in patients with Parkinson's disease? Parkinsonism Relat Disord. 2008;14(3):243-5. doi: 10.1016/j.parkreldis.2007.08.003. [PubMed: 17892967].

12. Ali GN, Wallace KL, Schwartz R, DeCarle DJ, Zagami AS, Cook IJ. Mechanisms of oral-pharyngeal dysphagia in patients with Parkinson's disease. Gastroenterology. 1996;110(2):383-92. doi: 10.1053/gast.1996.v110.pm8566584. [PubMed: 8566584].

13. Goldman JG, Vernaleo BA, Camicioli R, Dahodwala N, Dobkin RD, Ellis T, et al. Cognitive impairment in Parkinson's disease: A report from a multidisciplinary symposium on unmet needs and future directions to maintain cognitive health. NPJ Parkinsons Dis. 2018;4:19. doi: 10.1038/s41531-018-0055-3. [PubMed: 29951580]. [PubMed Central: PMC6018742]

14. Olchik MR, Ghisi M, Winckler PB, Ayres A. Correlation between cognitive aspects and dysphagia in patients with amyotrophic lateral sclerosis: Preliminary report. Int J Clin Med. 2017;8(12):680-91. doi: 10.4236/ijcm.2017.812063.

15. Kim JS, Youn J, Suh MK, Kim TE, Chin J, Park S, et al. Cognitive and motor aspects of Parkinson's disease associated with dysphagia. Can J Neurol Sci. 2015;42(6):395-400. doi: 10.1017/cjn.2015.304. [PubMed: 26551089].

16. Schroeder MF, Daniels SK, McClain M, Corey DM, Foundas AL. Clinical and cognitive predictors of swallowing recovery in stroke. J Reha- bil Res Dev. 2006;43(3):301-10. doi:10.1682/jrrd.2004.12.0154.[PubMed: 17041816].

17. Yang EJ, Kim KW, Lim JY, Paik NJ. Relationship between dysphagia and mild cognitive impairment in a community-based elderly cohort: The Korean longitudinal study on health and aging.J Am Geriatr Soc. 2014;62(1):40-6. doi: 10.1111/jgs.12606. [PubMed: 25180377].

18. Winchester J, Winchester CG. Cognitive dysphagia and effectively managing the five systems. Perspect Gerontol. 2015;20(3):116-32. doi: 10.1044/gero20.3.116.

19. Jo SY, Hwang JW, Pyun SB. Relationship between cognitive function and dysphagia after stroke. Ann Rehabil Med. 2017;41(4):564-72. doi: 10.5535/arm.2017.41.4.564. [PubMed: 28971040]. [PubMed Central: PMC5608663].

20. Hughes AJ, Ben-Shlomo Y, Daniel SE, Lees AJ. UK Parkinson's disease society brain bank clinical diagnostic criteria. J Neurol Neurosurg Psychiatr.1992;55(181). e4.

21. Kalf JG, Smit AM, Bloem BR, Zwarts MJ, Munneke M. Impact of drooling in Parkinson's disease. J Neurol. 2007;254(9):1227-32. doi: 10.1007/s00415-007-0508-9. [PubMed: 17671806].

22. Bakhtiyari J, Salmani M, Noruzi R, Sarraf P, Barzegar E, Mirmohammadkhani M. Translation, cross-cultural adaptation, validation and reliability of the northwestern dysphagia patient check sheet (NDPCS) in Iran. Iran J Otorhinolaryngol. 2018;30(97):97-101. [PubMed: 29594076]. [PubMed Central: PMC5866488].

23. Ansari NN, Naghdi S, Hasson S, Valizadeh L, Jalaie S. Validation of a mini-mental state examination (MMSE) for the Persian population: A pilot study. Appl Neuropsychol. 2010;17(3):190-5. doi: 10.1080/09084282.2010.499773. [PubMed: 20799110].

24. Nienstedt JC, Buhmann C, Bihler M, Niessen A, Plaetke R, Gerloff C, et al. Drooling is no early sign of dysphagia in Parkinson's disease. Neurogastroenterol Motil. 2018;30(4). e13259. doi: 10.1111/nmo.13259. [PubMed: 29178420].

25. Manor Y, Giladi N, Cohen A, Fliss DM, Cohen JT. Validation of a swallowing disturbance questionnaire for detecting dysphagia in patients with Parkinson's disease. Mov Disord. 2007;22(13):1917-21. doi: 10.1002/mds.21625. [PubMed: 17588237].

26. Solari N, Bonito-Oliva A, Fisone G, Brambilla R. Understanding cognitive deficits in Parkinson's disease: lessons from preclinical animal models. Learn Mem. 2013;20(10):592-600. doi: 10.1101/lm.032029.113. [PubMed: 24049188].

27. Rana AQ, Khondker S, Kabir A, Owalia A, Khondker S, Emre M. Impact of cognitive dysfunction on drooling in Parkinson's disease. Eur Neurol. 2013;70(1-2):42-5. doi: 10.1159/000348571. [PubMed: 23711510].

28. Rana AQ, Yousuf MS, Awan N, Fattah A. Impact of progression of Parkinson's disease on drooling in various ethnic groups. Eur Neurol. 2012;67(5):312-4. doi: 10.1159/000336054. [PubMed: 22517489].

29. Kalf JG. Management of dysphagia and drooling in patients with Parkinson's disease. Neurodegener Dis Manage. 2013;3(1):71-9. doi: 10.2217/nmt.12.77.

30. Nicaretta DH, de Rosso AL, Maliska C, Costa MM. Scintigraphic analysis of the parotid glands in patients with sialorrhea and Parkinson's disease. Parkinsonism Relat Disord. 2008;14(4):338-41. doi: 10.1016/j.parkreldis.2007.07.008. [PubMed:17855148].

31. Johnson J, Evatt ML, Chaudhuri KR. Sialorrhoea in Parkinson's disease. Non-Motor Symptoms Parkinson's Dis. 2014:315-22. doi: 10.1093/med/9780199684243.003.0021.

32. Johnston BT, Li Q, Castell JA, Castell DO. Swallowing and esophageal function in Parkinson's disease. Am J Gastroenterol. 1995;90(10):17416. [PubMed: 7572887].

33. Reynolds H, Miller N, Walker R. Drooling in Parkinson's disease: Evidence of a role for divided attention. Dysphagia. 2018;33(6):809-17. doi: 10.1007/s00455-018-9906-7. 